\title{
Preparation of $\alpha$-damascone
}

\author{
Junyi Hu${ }^{1}$, Aiqiang Guo ${ }^{1}$, Yingqian $\mathrm{Xu}^{1 *}$, Changsheng $\mathrm{Pan}^{2}$, Xingfu Wei ${ }^{1}$, Longlong Wang ${ }^{1}$, \\ Guoyong Xiao ${ }^{1}$
}

\author{
${ }^{1}$ University of Science and Technology Liaoning, School of Chemical Engineering, Liaoning Anshan, 114051, P. R. China \\ ${ }^{2}$ Jijia Geen Bilding Tchnology co., LTD, Liaoning Anshan, 114051, P. R. China \\ *Corresponding author: e-mail: xuyingqian@ustl.edu.cn
}

\begin{abstract}
$\alpha$-Damascone is widely used in perfumes. However, the manufacture of $\alpha$-damascone remains challenging owing to the limitations of current production processes. Herein, $\alpha$-damascone was successfully synthesized from $\alpha$-ionone using a new route involving only four steps, namely oximization, epoxidation, dehydration, and reduction. The total yield was $54.9 \%$ with a final chemical purity of $97 \%$ (by GC). Only water, cyclohexane, and ethanol were used in the reactions except in the purification step, and all solvents could be recycled. The structures of the intermediates and target compound were identified by ${ }^{1} \mathrm{H}$ NMR and ${ }^{13} \mathrm{C}$ NMR analyses and MS experiments. This route is a simple and successful method for the industrial preparation of $\alpha$-damascone.
\end{abstract}

Keywords: $\alpha$-damascone, $\alpha$-ionone, preparation.

\section{INTRODUCTION}

Owing to the delicate fragrance it gives to certain roses, fruits, and nicotiana tabacum ${ }^{1}, \alpha$-damascone is used as a flavoring agent in high-end cosmetics, foods, and other applications. It is also a rare spice that has been developed in recent decades. Accordingly, $\alpha$-damascone has received much research attention recently. $\alpha$-Damascone can be extracted from black tea ${ }^{2}$ or tobacco ${ }^{3}$. However, the amount of $\alpha$-damascone that can be extracted from natural sources is low, and market demand remains far from being met owing to its complex synthesis. Therefore, the current price of $\alpha$-damascone is high, resulting in limited applications.

The synthesis of $\alpha$-damascone in several reaction steps has previously been reported from $\alpha$-ionone, an inexpensive and readily available starting material. $\alpha$-Damascone was successfully synthesized by Ohloff ${ }^{4}$ in the early 1970s in three steps using a Wharton rearrangement reaction, but the result was poor owing to numerous cyclization byproducts. Serra and Fuganti ${ }^{5}$ utilized a base-mediated elimination of 7-oxy-dihydroionol acetate to obtain $\alpha$-damascone. Boulin, B. ${ }^{6}$, Luis A.S. ${ }^{7}$, Gosselin, P. ${ }^{8}$, also reported methods of preparation of $\alpha$-Damascone respectively. However, the reaction conditions were harsh and expensive, and the yield was low. Another synthetic route was reported ${ }^{9}$ involving transforming an oxime into an isoxazole derivative and $\alpha$-damascone was obtained via a reduction reaction. However, preparation of the isoxazole derivative consumed huge amounts of iodine reagents, which was costly, and presented decolorization problems. We now report a feasible new route, involving the epoxidation of an oxime, followed by isoxazole preparation via dehydration with strong acid, as shown in Scheme 1, to avoid the aforementioned synthetic problems.

\section{EXPERIMENTAL}

General experimental procedures. NMR analyses were performed using an Avance 500 instrument (Bruker, USA) at 500 and $125 \mathrm{MHz}$ for ${ }^{1} \mathrm{H}$ and ${ }^{13} \mathrm{C}$ spectra, respectively. Spectra were recorded in deuterated solvents containing tetramethylsilane as an internal reference standard. ESI-MS data were obtained on an Agilent
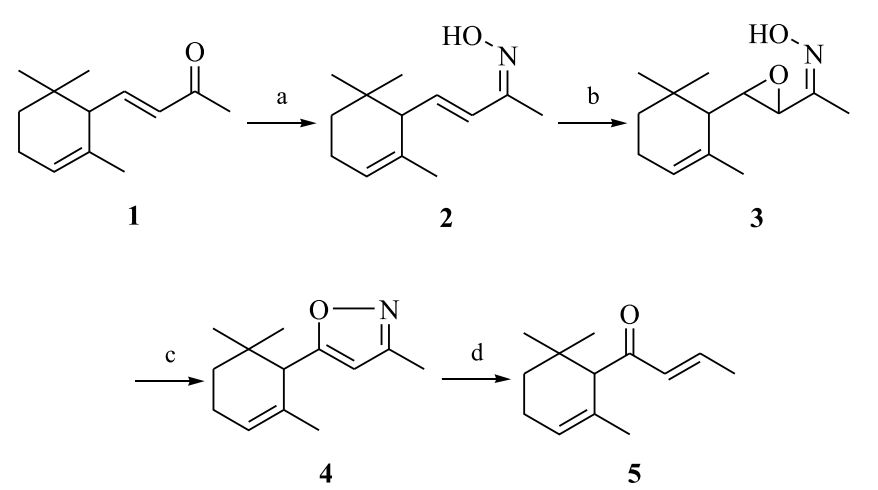

Scheme 1. Synthesis of $\alpha$-damascone

Reagents and conditions: a. $\mathrm{NH}_{2} \mathrm{OH} \cdot \mathrm{HCl}, \mathrm{NaOAc}$, $\mathrm{EtOH} / \mathrm{H}_{2} \mathrm{O}, 55^{\circ} \mathrm{C}, 99.6 \%$; b. $\mathrm{H}_{2} \mathrm{O}_{2} / \mathrm{H}_{2} \mathrm{O}, \mathrm{CTAOH}, \mathrm{LiOH}$, $\mathrm{H}_{2} \mathrm{O}, 15^{\circ} \mathrm{C}, 96.1 \%$; c. $\mathrm{HCl} / \mathrm{H}_{2} \mathrm{O}$, cyclohexane, $35^{\circ} \mathrm{C}$, $79.5 \%$; d. Na, $\mathrm{C}_{2} \mathrm{H}_{5} \mathrm{OH}$, cyclohexane, $35^{\circ} \mathrm{C}, 72.4 \%$

6530 Q-TOF instrument (Agilent, Singapore). $\alpha$-Ionone (1) was purchased from Haihang Industry Co., Ltd.

$\boldsymbol{\alpha}$-Ionone oxime (2). Hydroxylamine hydrochloride (3.8 g, $0.06 \mathrm{~mol})$ and sodium acetate $(6.4 \mathrm{~g}, 0.07 \mathrm{~mol})$ were dissolved in water $(15 \mathrm{~mL})$ and the solution was added dropwise to a solution of $\alpha$-ionone (1) $(10.0 \mathrm{~g}, 0.052 \mathrm{~mol})$ in ethanol $(20 \mathrm{~mL})$. The reaction was then stirred for 4 $\mathrm{h}$ at $55^{\circ} \mathrm{C}$. The mixture was cooled to room temperature, diluted with water $(60 \mathrm{~mL})$, and extracted with EtOAc $(80$ $\mathrm{mL} \times 3)$. The organic phase was washed with saturated $\mathrm{NaHSO}_{3}(100 \mathrm{~mL})$, dried with $\mathrm{MgSO}_{4}$, and concentrated in vacuo to afford $2(9.3 \mathrm{~g}, 99.6 \%$ yield, $98 \%$ chemical purity by GC, a mixture of two cis/trans isomers) as a sticky orange oil. $\mathrm{C}_{13} \mathrm{H}_{21} \mathrm{NO}$; mass spectrum (ESI), $m / z 207[\mathrm{M}]^{+}$. These diastereoisomers were used in the next steps without purification or separation.

Isomer 2a ${ }^{1} \mathrm{H}$ NMR $\left(500 \mathrm{MHz}, \mathrm{CDCl}_{3}\right) \delta 9.86(\mathrm{~s}$, $1 \mathrm{H}), 6.12(\mathrm{~d}, J=15.8 \mathrm{~Hz}, 1 \mathrm{H}), 5.86(\mathrm{dd}, J=15.7,9.5$ $\mathrm{Hz}, 1 \mathrm{H}), 5.42(\mathrm{~s}, 1 \mathrm{H}), 2.22(\mathrm{~d}, J=9.4 \mathrm{~Hz}, 1 \mathrm{H}), 2.00$ $(\mathrm{s}, 5 \mathrm{H}), 1.55(\mathrm{~s}, 3 \mathrm{H}), 1.51-1.34(\mathrm{~m}, 1 \mathrm{H}), 1.18(\mathrm{~d}, J=$ $13.2 \mathrm{~Hz}, 1 \mathrm{H}), 0.89$ (s, 3H), 0.81 (s, 3H). ${ }^{13} \mathrm{C}$ NMR $(125$ $\left.\mathrm{MHz}, \mathrm{CDCl}_{3}\right) \delta_{\mathrm{C}} 155.7,136.5,132.8,127.9,120.9,53.9$, $31.7,30.9,28.2,27.0,26.3,22.2,9.3$.

Isomer $2 \mathbf{b}{ }^{1} \mathrm{H}$ NMR $\left(500 \mathrm{MHz}, \mathrm{CDCl}_{3}\right) \delta 8.32(\mathrm{~s}, 1 \mathrm{H})$, $6.84(\mathrm{~d}, J=16.0 \mathrm{~Hz}, 1 \mathrm{H}), 6.17-5.78(\mathrm{~m}, 1 \mathrm{H}), 5.44(\mathrm{~s}, 1 \mathrm{H})$, $2.25(\mathrm{t}, J=12.5 \mathrm{~Hz}, 1 \mathrm{H}), 2.10-1.82(\mathrm{~m}, 5 \mathrm{H}), 1.56(\mathrm{~s}, 3 \mathrm{H})$, $1.47-1.36$ (m, 1H), 0.98 (d, $J=19.4 \mathrm{~Hz}, 1 \mathrm{H}), 0.90(\mathrm{~s}, 3 \mathrm{H})$, 
$0.81(\mathrm{~s}, 3 \mathrm{H}) \cdot{ }^{13} \mathrm{C}$ NMR $\left(125 \mathrm{MHz}, \mathrm{CDCl}_{3}\right) \delta_{\mathrm{C}} 152.4,140.8$, 132.4, 121.2, 119.9, 53.9, 31.7, 30.9, 27.0, 26.3, 22.4, 22.2, 9.3.

$\boldsymbol{\alpha}$-Ionone oxime epoxide (3). $\alpha$-Ionone oxime 2 (5.1 g, $0.025 \mathrm{~mol}$, a mixture of two cis/trans isomers obtained in step 1) and $10 \mathrm{wt} \% \mathrm{CTAOH}$ aqueous solution (3.7 $\mathrm{mL})$ were dissolved in water $(70 \mathrm{~mL})$ at $0^{\circ} \mathrm{C} \cdot \mathrm{H}_{2} \mathrm{O}_{2}(35$ $\mathrm{mL}, 30 \%$ in water) and $\mathrm{LiOH}$ aqueous solution $(5 \mathrm{~mL}$, $6 \mathrm{~mol} / \mathrm{L}$ ) were mixed and was slowly added at below $15^{\circ} \mathrm{C}$. After $15 \mathrm{~h}$, the reaction mixture was extracted with $\mathrm{CH}_{2} \mathrm{Cl}_{2}(50 \mathrm{~mL} \times 3)$, dried with $\mathrm{MgSO}_{4}$, and condensed to provide 3 (4.9 g, 96.1\% yield, 97\% chemical purity by $\mathrm{GC}$, a mixture of multiple diastereoisomers) as a sticky oil. $\mathrm{C}_{13} \mathrm{H}_{21} \mathrm{NO}_{2}$; mass spectrum (ESI), $\mathrm{m} / \mathrm{z}$ $223[\mathrm{M}]^{+}$. These diastereoisomers were used in the next steps without purification or separation.

Isomer 3a: ${ }^{1} \mathrm{H} \mathrm{NMR}\left(500 \mathrm{MHz}, \mathrm{CDCl}_{3}\right) \delta 5.52(\mathrm{~s}, 1 \mathrm{H})$, 3.42 (m, 1H), 2.84 (dd, $J=15.9,8.7 \mathrm{~Hz}, 1 \mathrm{H}), 2.04$ (s, $3 \mathrm{H}), 1.89(\mathrm{~m}, 1 \mathrm{H}), 1.80(\mathrm{~m}, 1 \mathrm{H}), 1.71(\mathrm{~d}, J=5.1 \mathrm{~Hz}$, $3 \mathrm{H}), 1.56(\mathrm{~m}, 1 \mathrm{H}), 1.42(\mathrm{~d}, J=8.7 \mathrm{~Hz}, 1 \mathrm{H}), 1.25(\mathrm{~m}$, 1H), 1.11 (s, 3H), 0.95 (s, 3H). ${ }^{13} \mathrm{C}$ NMR (125 MHz, $\left.\mathrm{CDCl}_{3}\right) \delta_{\mathrm{C}} 155.4,130.3,123.5,56.4,56.1,51.7,32.6,31.9$, 31.3, 26.7, 23.0, 22.4, 7.6.

Isomer 3b: ${ }^{1} \mathrm{H}$ NMR $\left(500 \mathrm{MHz}, \mathrm{CDCl}_{3}\right) \delta 5.52(\mathrm{~s}, 1 \mathrm{H})$, $3.43(\mathrm{~s}, 1 \mathrm{H}), 2.84(\mathrm{t}, J=12.4 \mathrm{~Hz}, 1 \mathrm{H}), 2.04(\mathrm{~s}, 3 \mathrm{H}), 1.83$ $(\mathrm{m}, 1 \mathrm{H}), 1.71(\mathrm{~d}, J=5.7 \mathrm{~Hz}, 3 \mathrm{H}), 1.55(\mathrm{~m}, 1 \mathrm{H}), 1.42(\mathrm{~d}$, $J=8.8 \mathrm{~Hz}, 1 \mathrm{H}), 1.40-1.12(\mathrm{~m}, 2 \mathrm{H}), 1.11(\mathrm{~s}, 3 \mathrm{H}), 0.94$ (s, 3H). ${ }^{13} \mathrm{C}$ NMR $\left(125 \mathrm{MHz}, \mathrm{CDCl}_{3}\right) \delta_{\mathrm{C}} 155.4,130.3$, 123.5, 56.4, 56.0, 51.7, 31.9, 31.3, 26.7, 26.4, 23.1, 22.4, 7.6.

a-Ionone isoxazole (4). $\mathrm{HCl}(1.5 \mathrm{~mL}, 35 \%$ in water $)$ was added dropwise to a solution of $\mathbf{3}(2.0 \mathrm{~g}, 0.009$ mol) in cyclohexane $(20 \mathrm{~mL})$ at $35^{\circ} \mathrm{C}$. After addition was complete, the mixture was stirred under reflux for $6 \mathrm{~h}$. The reaction mixture was then diluted with water $(50 \mathrm{~mL})$, extracted with $\mathrm{CH}_{2} \mathrm{Cl}_{2}(70 \mathrm{~mL} \times 3)$, neutralized with $10 \% \mathrm{NaOH}$ aqueous solution, and the organic layer was collected and condensed to provide 4 (1.59 g, $79.5 \%$ yield, $92 \%$ chemical purity (GC)) as a sticky oil. $\mathrm{C}_{13} \mathrm{H}_{19} \mathrm{NO}$; mass spectrum (ESI), $m / z 205[\mathrm{M}]^{+}$.

${ }^{1} \mathrm{H}$ NMR $\left(500 \mathrm{MHz} \mathrm{CDCl}_{3}\right) \delta 5.72(\mathrm{~s}, 1 \mathrm{H}), 5.50(\mathrm{~s}$, $1 \mathrm{H}), 2.91(\mathrm{~s}, 1 \mathrm{H}), 2.20(\mathrm{~s}, 3 \mathrm{H}), 2.03(\mathrm{~m}, 2 \mathrm{H}), 1.50(\mathrm{~s}$, $3 \mathrm{H}), 1.15(\mathrm{~m}, 2 \mathrm{H}), 0.94(\mathrm{~s}, 3 \mathrm{H}), 0.68(\mathrm{~s}, 3 \mathrm{H}) .{ }^{13} \mathrm{C} \mathrm{NMR}$ $\left(125 \mathrm{MHz}, \mathrm{CDCl}_{3}\right) \delta_{\mathrm{C}} 173.9,158.7,130.8,122.2,102.5$, 48.8, 32.2, 32.1, 30.2, 27.3, 25.9, 22.2, 10.8 .

$\boldsymbol{\alpha}$-Damascone (5). Sodium (1.1 g, $0.5 \mathrm{~mol})$ was added to a solution of $\alpha$-ionone isoxazole $4(2.1 \mathrm{~g}, 0.010 \mathrm{~mol})$ in cyclohexane $(40 \mathrm{~mL})$ and absolute ethanol $(5 \mathrm{~mL})$ at $35^{\circ} \mathrm{C}$. When the sodium was completely dissolved, the mixture was heated at reflux for $8 \mathrm{~h}$. $\mathrm{NH}_{4} \mathrm{Cl}(16 \mathrm{~mL}, 20 \%$ in water) were then added to the reaction mixture, which was then cooled to room temperature. The mixture was extracted with EtOAc $(70 \mathrm{~mL} \times 3)$ and the organic phase was washed with saturated $\mathrm{NaHCO}_{3}(50 \mathrm{~mL})$, dried with $\mathrm{MgSO}_{4}$, concentrated in vacuo to give the crude product. After distillation, $\alpha$-damascone (1.52 g, 72.4\% yield, $97 \%$ chemical purity (GC)) was obtained as a sticky oil. $\mathrm{C}_{13} \mathrm{H}_{20} \mathrm{O}$; mass spectrum (ESI), $m / z 192[\mathrm{M}]^{+}$.

${ }^{1} \mathrm{H}$ NMR $\left(500 \mathrm{MHz} \mathrm{CDCl}_{3}\right) \delta 6.85(\mathrm{~m}, 1 \mathrm{H}), 6.30(\mathrm{~d}$, $J=15.4 \mathrm{~Hz}, 1 \mathrm{H}), 5.59(\mathrm{~m}, 1 \mathrm{H}), 2.88(\mathrm{~s}, 1 \mathrm{H}), 2.07(\mathrm{dt}$, $J=20.3,15.3 \mathrm{~Hz}, 2 \mathrm{H}), 1.87(\mathrm{t}, J=11.4 \mathrm{~Hz}, 3 \mathrm{H}), 1.68$ $(\mathrm{m}, 1 \mathrm{H}), 1.54(\mathrm{~m}, 3 \mathrm{H}), 1.14(\mathrm{dt}, J=19.8,10.0 \mathrm{~Hz}, 1 \mathrm{H})$, 0.93 (s, 3H), 0.84 (s, 3H). ${ }^{13} \mathrm{C}$ NMR $\left(125 \mathrm{MHz}, \mathrm{CDCl}_{3}\right)$ $\delta_{\mathrm{C}} 201.6,141.6,131.6,129.9,122.9,60.7,31.8,30.7,27.4$, 27.3, 22.6, 22.0, 17.5.

\section{RESULTS AND DISCUSSION}

In this synthesis, $\alpha$-ionone oxime was prepared from $\alpha$-ionone and hydroxylamine hydrochloride in water and ethanol. And in the key epoxidation reaction (b), water, which is an important environmentally friendly solvent, was used as the solvent instead of an organic solvent ${ }^{\mathbf{1 0}}$. Furthermore, hydrogen peroxide was used as oxidant with cetyltrimethylammonium hydroperoxide $(\mathrm{CTAOH})$ as the catalyst for the epoxidation reaction so only water was the byproduct for this reaction. The diastereomeric ratios was not analyzed because all of the diastereoisomers of compound $\mathbf{2}$ and $\mathbf{3}$ would product the same product (compound 4) after the next steps.

After $\alpha$-ionone oxime epoxide was converted to $\alpha$-ionone isoxazole in cyclohexane, the final reduction step was achieved using sodium in ethanol, which greatly reduced the reaction time, number of steps, and operational costs. The overall yield was $54.9 \%$, which is higher than those reported (from $23.3 \%$ to $41.5 \%$ ).

\section{ACKNOWLEDGMENTS}

This project was supported financially by the First-Class Discipline Group of the University of Science and Technology Liaoning. We thank Simon Partridge, $\mathrm{PhD}$, from Liwen Bianji, Edanz Editing China (www.liwenbianji.cn/ ac), for editing the English text of a draft of this manuscript.

\section{LITERATURE CITED}

1. Anthony, J.N.B., Stephen, W.P. \& John, S.S. (1983) A damascone derivative from Nicotiana tabacum. Phytochemistry. 22(2), 613-614. DOI: 10.1016/0031-9422(83)83068-4.

2. Kenji, M. \& Masayasu, A. (1993) Synthesis of (S)- $\alpha-$ damascone. Tetrahedron. 49(9), 1871-1878. DOI: 10.1016/ S0040-4020(01)80543-3.

3. Werkhoff, P., Bretschneider, W. \& Güntert, M., et al. (1991) Chirospecific analysis in flavor and essential oil chemistry Part B. Direct enantiomer resolution of frans- $\alpha$-ionone and trans$\alpha$-damascone by inclusion gas chromatography. Z. Lebensm.-Unters. Forsch. 192(2), 111-115. DOI: 10.1007/BF01202622.

4. Ohloff, G. \& Uhde, G. (2004) Über eine ungewöhnliche Cyclisationsreaktion bei der Umsetzung von $(+)$-Epoxy- $\alpha$ dihydrojonon mit Hydrazinhydrat. Helv. Chim. Acta. 53(3): 531-541. DOI: 10.1002/hlca.19700530309.

5. Stefano, S. \& Claudio, F. (2006) Synthesis of the enantiomeric forms of $\alpha$ - and $\gamma$-damascone starting from commercial racemic $\alpha$-ionone. Tetrahedron Asymmetry. 17(10), 1573-1580. DOI: 10.1016/j.tetasy.2006.05.024.

6. Boulin, B., Taran, M. \& Arreguy-San, M.B., et al. (2007) New Preparation Methods for $\alpha$-Damascone, $\gamma$-Damascone, and $\beta$-Damascenone using Pyronenes. Communications. 37(15), 2579-2591. DOI: 10.1080/00397910701462898.

7. Luis, A.S. \& Jean, L.L. (1992) A new synthesis of $\alpha$ - and $\beta$-damascones from the ionones. J. Org. Chem., 57(9), 2757-2760. DOI: $10.1021 /$ jo00035a045.

8. Gosselin, P. (1988) Acid catalyzed hydrolysis of a dienyl sulfide. Application to the synthesis of $\alpha$-damascone. Tetrahedron, 44(7), 1979-1990. DOI: 10.1002/chin.198831288.

9. Buechi, G. \& Vederas, J.C. (1972) Interchange of functionality in conjugated carbonyl compounds through isoxazoles. $J$. Am. Chem. Soc. 94(26), 9128-9132. DOI: 10.1021/ja00781a023.

10. Fioroni, G., Fringuelli, F. \& Pizzo, F., et al. (2003) Epoxidation of $\alpha, \beta$,-unsaturated ketones in water. An environmentally benign protocol. Green Chem. 5(4), 425-428. DOI: 10.1039/ b303883a. 